\title{
CONTRIBUIÇÃO AO ESTUDO DO COMPORTAMENTO DE PENEIRA VIBRATÓRIA TIPICAMENTE EMPREGADA EM UNIDADE DE TRATAMENTO DE FLUIDO DE PERFURAÇÃO
}

\author{
E. N. da MATTA ${ }^{1}$, C. A. P. CIPELLI ${ }^{1}$, M. M. GOMES ${ }^{1}$, F. S. GUERREIRO ${ }^{2}$, \\ R. GEDRAITE ${ }^{2}$, L. KUNIGK ${ }^{1}$ e W. de O. ASSIS ${ }^{1}$ \\ ${ }^{1}$ Centro Universitário do Instituto Mauá de Tecnologia, Coordenadoria de Pós-Graduação \\ ${ }^{2}$ UniversidadeFederal de Uberlândia,Faculdade de Engenharia Química \\ E-mail para contato: eduardonadaleto@ maua.br
}

\begin{abstract}
RESUMO - A recuperação do fluido de perfuração é importante na operação de perfuração de poços de petróleo, sob a ótica ambiental e econômica. Os equipamentos utilizados para a separação sólido-líquido são divididos em três grupos sequenciais: peneiras vibratórias, bateria de hidrociclones e centrífuga decantadora. $\mathrm{O}$ entendimento do comportamento do processo de separação nas peneiras pode contribuir para a melhoria operacional e aumento da vida útil das telas usadas. Este trabalho apresenta uma contribuição ao tema, abordando a definição e implantação de sistema de monitoração do tamanho de partículas e/ou nível de material em protótipo de peneira. O sistema foi desenvolvido com base na ferramenta LabVIEW $^{\mathrm{TM}}$ e foi capaz de identificar de forma satisfatória o tamanho de material particulado presente sobre a tela de separação. Os resultados obtidos sugerem que o sistema estudado apresenta boa aderência ao comportamento real do sistema, com desvios considerados aceitáveis para fins de controle de processo.
\end{abstract}

\section{INTRODUÇÃO}

A eficiência de uma unidade de tratamento de fluido de perfuração pode ser avaliada de duas maneiras, a saber: (i)- eficiênciana remoção de sólidos propriamente ditos e (ii)- eficiência na conservação de líquido. Quanto maior a fração de sólidos removidos, maior a eficiência. Quanto maior a fração de sólidos no efluente de descarte, melhor é a eficiência de remoção. Ambos os aspectos devem ser considerados. Um bom projeto de sistema de separação de sólidos leva em conta a instalação de vários tipos de equipamentos em série, tais como: peneiras, hidrociclones, mudcleaners e centrífugas decantadoras(ASME, 2004). É de suma importância observar que cada estágio de processamento possui um elevado grau de dependência com o seu predecessor, ou seja, para que um dado equipamento tenha uma eficiência adequada, o equipamento em série imediatamente antes deve estar operando de acordo com a sua capacidade. Casocontrário, haverá uma sobrecarga para o equipamentoseguinte, ocasionado uma perda de eficiência global da unidade de recuperação.

Deve ser ressaltado o fato de que as peneiras têm um papel fundamental na eficiência global do sistema, pois são os primeiros equipamentos que entram em contato com o fluido de perfuração 


\section{9 a 22 de outubro de 2014 \\ Florianópolis/SC}

quando este retorna do poço. $\mathrm{O}$ dimensionamento adequado da quantidade e qualidade das peneiras evita a sobrecarga dos hidrociclones (desareiadores e dessiltadores) e dos equipamentos seguintes.

No contexto da operação de uma unidade de tratamento de fluido de perfuração típica, as variáveis de processo que devem ser monitoradas e/ou controladas, são: distribuição do tamanho das partículas, densidade do fluido, viscosidade do fluido, vazão de fluidoe variação de volume do fluido na unidade de tratamento.

Algumas destas variáveis já são medidas com relativa facilidade. Dentre elas podem-se citar a densidade, a viscosidade e a variação de volume. Outras ainda requerem o emprego de elementos sensores não totalmente consolidados para medição contínua e em tempo real, tal como o analisador de tamanho de partículas (Lobato et al., 2011).

\section{CONSIDERAÇÕES SOBRE O COMPORTAMENTO DINÂMICO DA PENEIRA VIBRATÓRIA}

Um dos principais equipamentos para o funcionamento correto e adequado da unidade de tratamento do fluido de perfuração é a peneira. Sem um peneiramento adequado do fluido de perfuração neste estágio inicial de separação de sólidos, a eficiência e efetividade de todo o sistema fica seriamente comprometida.

As peneiras evoluíram de equipamentos pequenos e simples, capazes de processar apenas sólidos mais grosseiros, aos atuais modelos encontrados no mercado. As peneiras modernas de alto desempenho estão equipadas com motovibradores que produzem movimentos de vários tipos e permitem variadas inclinações da tela e são capazes de operar com telas tão finas quanto 200 mesh na maioria das vazões encontradas em poços.

O propósito da vibração é o transporte do cascalho para fora da tela e o aumento da capacidade de processamento de líquido. Esta ação causa uma rápida separação, contribuindo para reduzir a quantidade de líquido residual arrastado com os sólidos.

A identificação da dinâmica de uma peneira vibratória típica é feita levando-se em consideração as variáveis de processo apresentadas na sequiência e classificadas em variáveis de saída e de entrada do sistema. A principal variável de saída será a Distribuição de Tamanho de Partículas (PSD, em inglês), considerada para fins de identificação da dinâmica do equipamento como a variável a ser controlada no mesmo. As outras variáveis de entrada do sistema são: (i) a força "G" aplicada; (ii) a elevação da peneira; (iii) a língua de fluido sobre a tela da peneira e (iv) a vazão de fluido de processo alimentada.

Um dos principais desafios nesse processo é caracterizar o tamanho das partículas e material sólido nas correntes de entrada e de saída da peneira. Assim, é possível ajustar condições operacionais, tais como: a vazão do fluido, a frequência e amplitude de vibração da tela e a inclinação da tela de filtração para melhorar a eficiência do processo de retirada dos detritos e reduzir o seu 


\section{9 a 22 de outubro de 2014 \\ Florianópolis/SC}

desgaste (Guerreiro, 2012).

\section{IDENTIFICAÇÃO DA PSD POR PROCESSAMENTO DE IMAGEM}

A aplicação de técnicas de processamento de imagens no desenvolvimento de sistemas de visão computacional é uma área de pesquisa que recentemente tem produzido resultados de grande relevância para inúmeros segmentos da sociedade (Gonzales e Woods, 2000). Pode-se citar, por exemplo, aplicações de identificação e segmentação de objetos ou imagens humanas (Fernandes e Gomes, 2002), reconstrução 3D de cenas reais, aplicações de prototipagem rápida (RP RapidPrototyping) para produção de objetos com formas complexas a partir de dados tridimensionais, rastreamento em tempo real e navegação de robôs móveis ou veículos não tripulados (Crawford et al., 2004), controle de processos de manufatura ou sistemas industriais (Deniset al., 2009), dentre outros.

O reconhecimento e classificação de padrões é parte essencial em um sistema de análise de imagens, e tem por objetivo analisar determinado evento ou objeto e classificá-lo dentre várias categorias preestabelecidas.

Em muitas aplicações o reconhecimento de padrões baseia na análise dos pixels de imagens coloridas. Nesse caso, as imagens são representadas por matrizes de pixels, sendo que cada uma das matrizes representam uma das cores primárias no padrão RGB (Red, Green e Blue) ou ainda combinações dessas matrizes. Em outras aplicações, mesmo que a câmera utilizada não obtenha imagens coloridas, a identificação ainda assim pode ser realizada pela análise dos níveis de cinza. No caso específico da aplicação tratada nesse trabalho, o objetivo de identificar as dimensões do cascalhofoialcançado com eficiência usando-se imagens em tons de cinza (greyscale).

Algumas das principais técnicas utilizadas em aplicações de processamento de imagem digital onde o objetivo é a classificação a partir das dimensões do objeto incluem a binarização e a conectividade. A binarização da imagem consiste na transformação da imagem para níveis branco e preto puro. Para isso obtém-se um histograma onde são analisados os valores na escala greyscale obtidos para cada pixel da imagem e sua distribuição em termos de intensidade. Uma maneira de determinar os níveis da imagem binarizada (preto ou branco) e comparar os valores dos pixels da matriz greyscale utilizando um limiar (threshold). Quando um único limiar é utilizado para uma imagem inteira, o processo é conhecido como limiarização global. Quando o limiar muda ao longo da imagem, temos a limiarização variável. Se o limiar é variável e definido a partir das propriedades de uma vizinhança (por exemplo, a média dos pixels da vizinhança), temos a limiarização local (Gonzales e Woods, 2000). Uma das técnicas de limiarização de imagens é o algoritmo conhecido como filtro de Sobel. Trata-se de uma operação usada em processamento de imagens especialmente em algoritmos aplicados na detecção de contorno (Kimmelet al., 2005).

\section{MATERIAIS E MÉTODOS}

Foi utilizada na captura das imagens uma câmera IP (Internet Protocol) Feasso ${ }^{\mathrm{TM}}$ modelo fipcam03, com processador de 32 bits, sensor CMOS de alta sensibilidade e imagem VGA com resolução de 640 x 480 pixels. Esta câmera permite comunicação remota com um computador com 
rede 802.11 (Wi-Fi) e 802.3 (RJ45 10 base T). Ela foi instalada em um suporte de metal de modo a manter a distância entre a lente e a peneira, garantindo assim uniformidade na aquisição das imagens em diferentes situações de teste (Figura 1). A câmera foi preparada para o ambiente agressivo em que estará instalada na indústria, sendo exposta ao sol e à chuva, e no caso de plataformas de perfuração, ao sal carregado pelos ventos marinhos.

Na aplicação utilizou-se uma rede com protocolo TCP/IP (Transmission Control Protocol) para fazer a ligação entre o cliente FTP (File Transfer Protocol) presente na câmera e um servidor que será instalado no computador servidor. Com a utilização deste protocolo é possível fazer o envio dos arquivos presentes na câmera de modo relativamente seguro, com relativa imunidade à perda ou corrupção da informação. O modelo de comunicação FTP adotado é realizado como ilustrado na Figura 2.
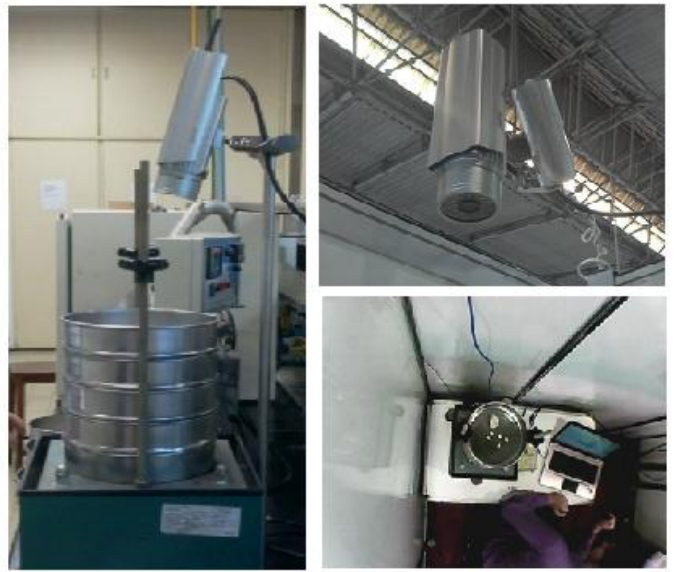

Figura 1 - Montagem para ensaios testes iniciais realizados em Matlab ${ }^{\mathrm{TM}}$

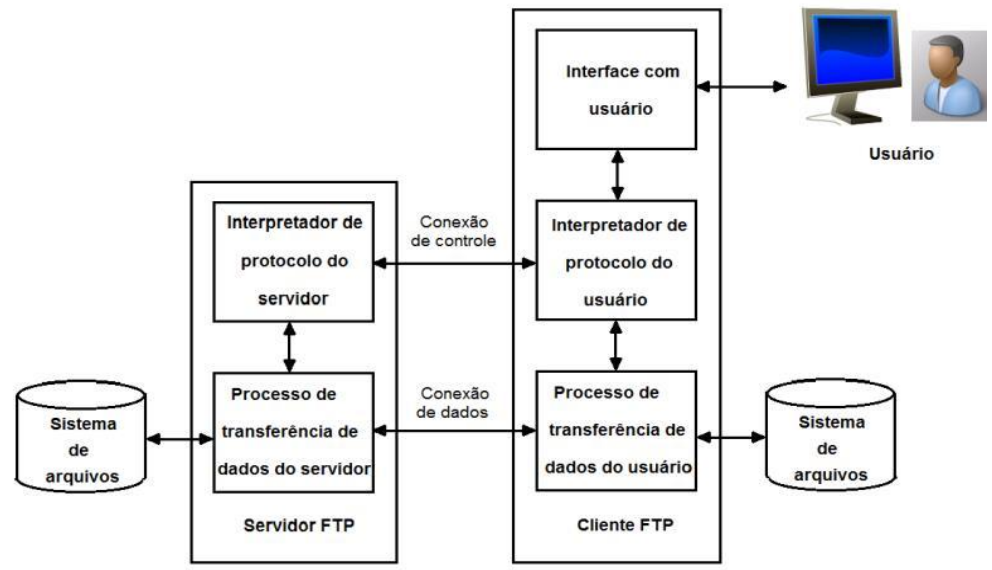

Figura 2 - Modelo de conexão FTP

A interface gráfica apresentada na Figura 3 foi desenvolvida utilizando o aplicativo NI Vision que integrado ao software LabVIEW ${ }^{\mathrm{TM}}$ da National Instruments. O aplicativo desenvolvido no software LabVIEW ${ }^{\mathrm{TM}}$ pode ser resumido pelo diagrama de blocos da Figura 4.

A interface gráfica inclui diversas funcionalidades, a saber: (i)- seleção da fonte das imagens a serem utilizadas (externas - capturadas por câmera - ou obtidas por meio de figuras armazenadas); (ii)- calibração da imagem utilizando um padrão, podendo opcionalmente realizar a manipulação de contraste, brilho e gama da imagem; (iii)- processamento incluindo conversão de imagens para tons de cinza, binarização e seleção de filtros; (iv)- eliminação de conglomerados de rochas acumulados nas bordas e interferências utilizando fator de enlogação; (v)- determinação do número de partículas e cálculo da área de cada uma delas, bem como da área média; (vi)- opção de utilização de sistema de monitoramento remoto utilizando página da Web. 

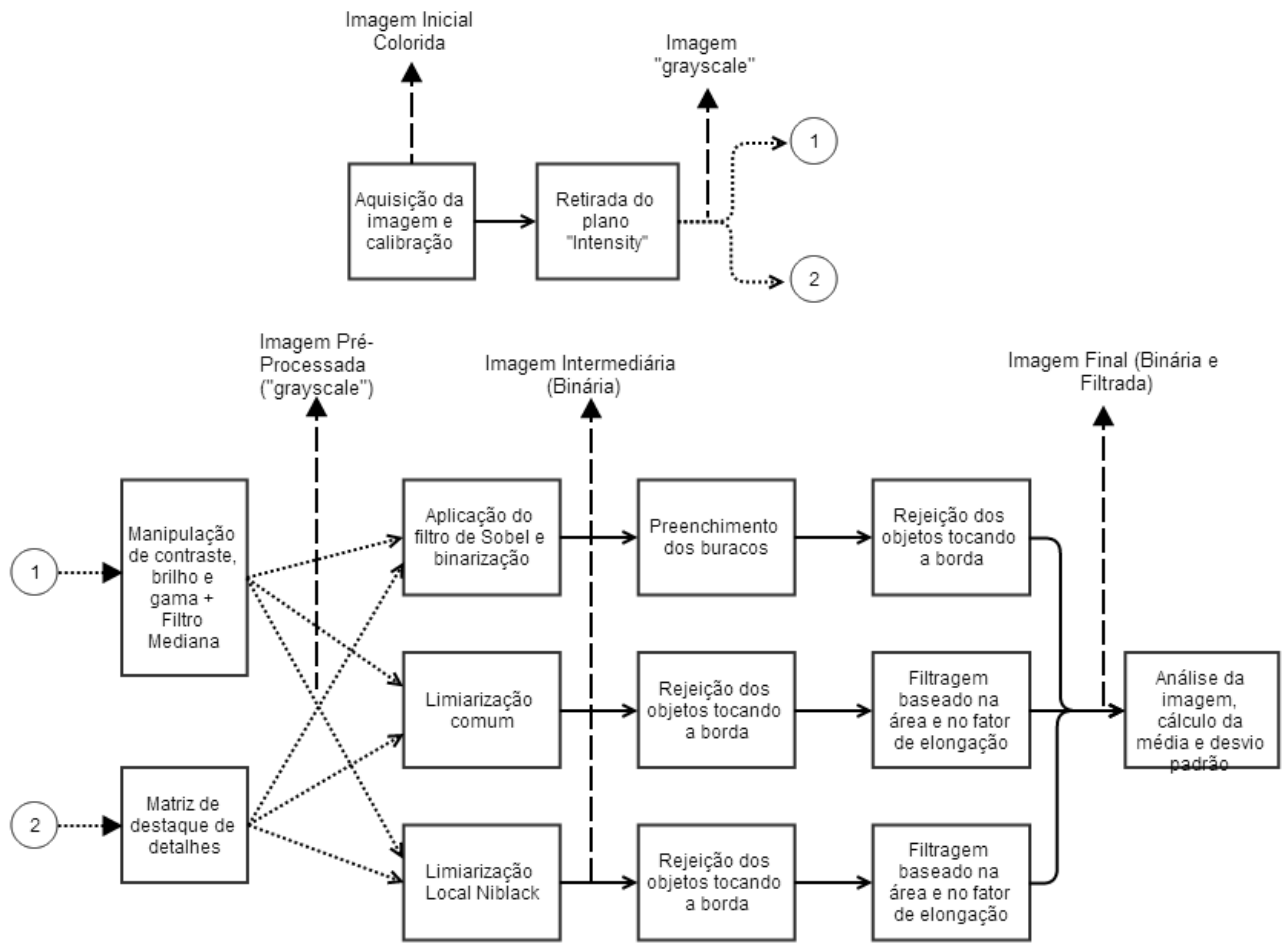

Figura 3 - Interface gráfica para o usuário

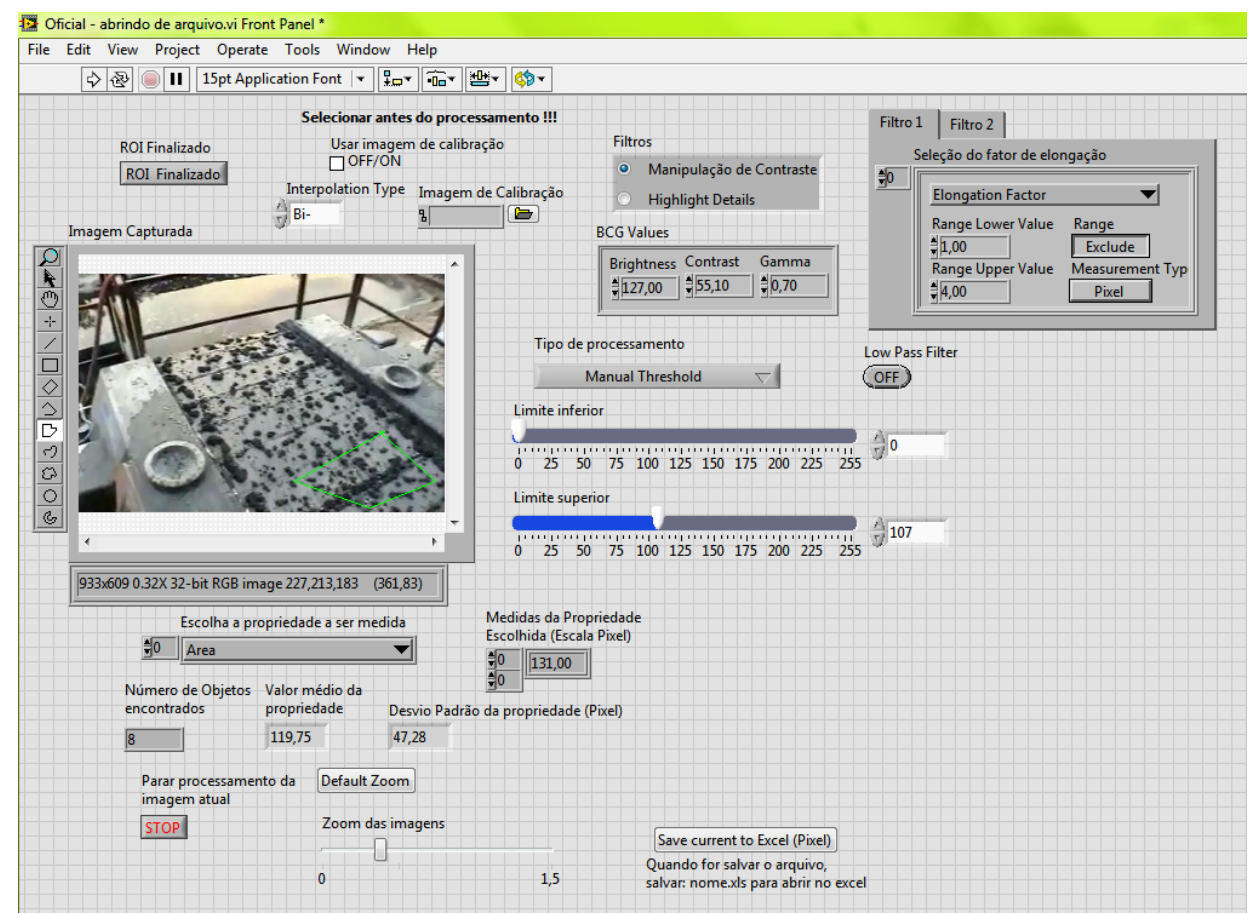

Figura 4 - Algoritmo completo do sistema de processamento de imagem 


\section{RESULTADOS EXPERIMENTAIS}

O sistema desenvolvido foi utilizado para processar imagens similares às capturadas em sistemas reais. A Figura 5 mostra um exemplo de imagens utilizadas nos testes do algoritmo em $\mathrm{LabVIEW}^{\mathrm{TM}}$ com destaque para a região de interesse de uma peneira vibratória típica.

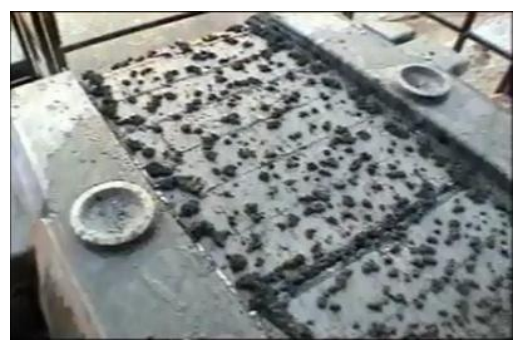

(a) Imagem real

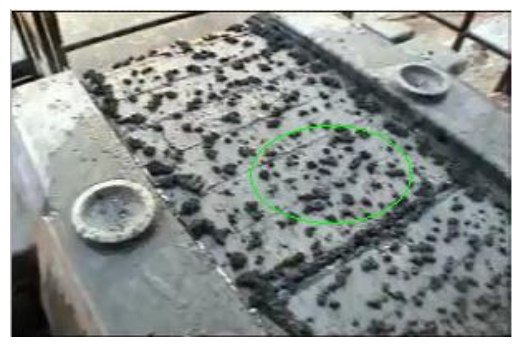

(b) Imagem com delimitação da região de interesse

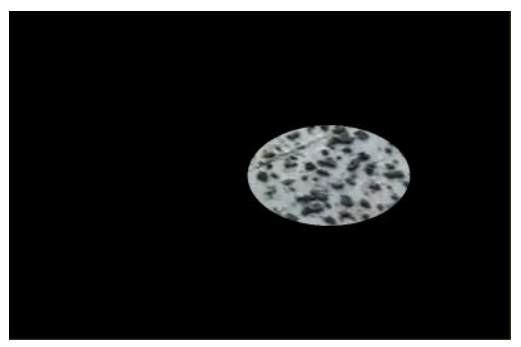

(c) Destaque da região de interesse

Figura 5. Imagem utilizada nos testes

A Figura 6 apresenta alguns resultados obtidos utilizando as seguintes combinações das funcionalidades disponíveis no algoritmo: (a) binarização aplicando algoritmo de destaque de detalhes e filtro de Sobel; (b) binarização aplicando algoritmo de destaque de detalhes,limiarização global e filtro de partículas; (c) binarização aplicando algoritmo de destaque de detalhes, limiarização local com filtro do tipo Niblack e filtro de partículas; (d) binarização com manipulação de contraste, brilho e gama, limiarização global e filtro de partículas.

A Tabela 1 apresenta um resumo dos resultados obtidos. Observa-se que, embora o filtro de Sobel seja eficiente na detecção de bordas, a identificação de partículas produziu resultado nulo. Isso ocorreu porque após o preenchimento resulta em partículas com grandes dimensões, sendo eliminadas pelo filtro de área ou elongação. Contudo, desprezando esses resultados, os outros métodos apresentaram excelentes resultados, inclusive com relativa precisão no cálculo da área das partículas identificadas. $\mathrm{O}$ erro apresentado na Tabela $1 \mathrm{diz}$ respeito ao número de objetos identificados em relação ao número real de objetos presentes sobre a tela da peneira estudada.

Tabela 1 - Resultados obtidos no software LabVIEW ${ }^{\mathrm{TM}}$ para as imagens da Figura 6

\begin{tabular}{ccc} 
Imagem & $\mathrm{N}^{\mathrm{o}}$ de Objetos Identificados & Erro (\%) \\
\hline $\mathrm{a}$ & 0 & 100 \\
$\mathrm{~b}$ & 26 & 0 \\
$\mathrm{c}$ & 26 & 0 \\
$\mathrm{~d}$ & 24 & 7,7 \\
\hline
\end{tabular}




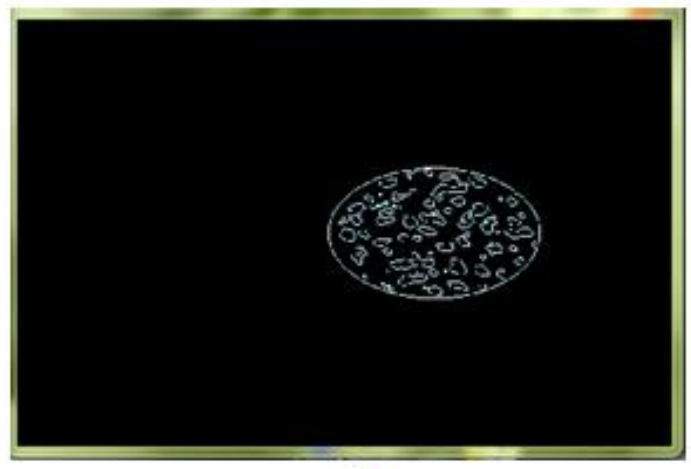

(a)

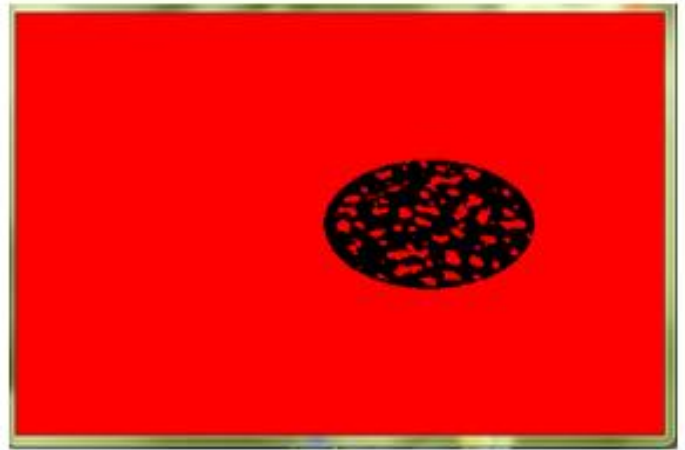

(c)

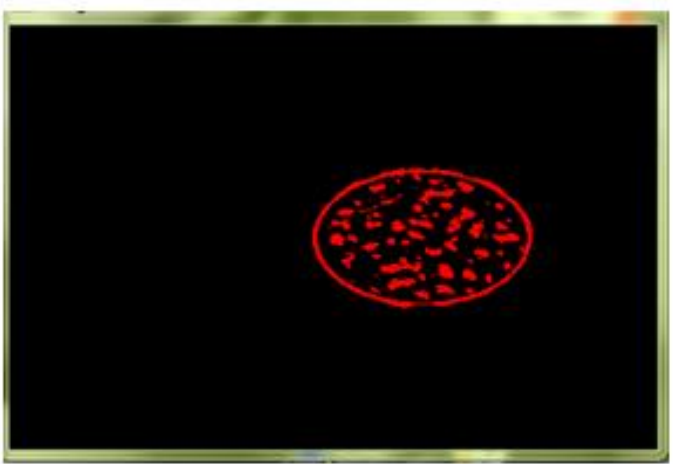

(b)

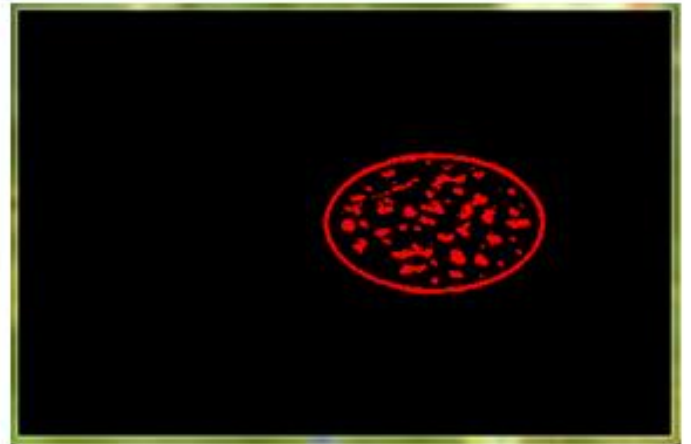

(d)

Figura 6. Resultados obtidos no aplicativo em LabVIEW ${ }^{\mathrm{TM}}$, considerando a binarização aplicando:(a)- filtro de Sobel; (b)- limiarização global e filtro de partículas; (c)- limiarização local com filtro do tipo Niblack e filtro de partículas e (d)- com manipulação de contraste, brilho e gama, limiarização global e filtro de partículas

\section{CONCLUSÕES}

O trabalho permitiu o desenvolvimento de um algoritmo de processamento de imagem que inclui diversas funcionalidades e que permite caracterizar de forma adequada e coerente a granulometria do material sólido particulado presente no fluido de perfuração de poços de petróleo, mesmo com relativa alteração de iluminação do local. Embora eficiente, o algoritmo apresentou as seguintes limitações: (i) imprecisão nas medições quando há acúmulo de material particulado, sobretudo nas bordas da peneira de vibração e (ii) intervenção do operador para selecionar a melhor combinação de funções e filtros, visando garantir melhor eficiência no algoritmo.

\section{AGRADECIMENTOS}

Os autores agradecem ao IMT,à UFU, e à FAPEMIG (Fundação de Amparo à Pesquisa do 
Estado de Minas Gerais) pelos recursos concedidos e apoio em pesquisas no Projeto de Participação Coletiva em Eventos Técnico-Científicos (PCE-00082-14).

\section{REFERÊNCIAS}

ASME - Shale Shaker Committee of the American Society of Mechanical Engineer Drilling Fluid Processing Handbook.EUA: Gulf Professional Publishing, 2004.

CRAWFORD, B. G.; DOWNING, D. R. Design and Evaluation of an Autonomous, Obstacle Avoiding, Flight Control System Using Simulated Visual Sensors. 2004. In:Anais do AIAA $2^{\text {nd }}$ Unmanned Unlimited Technical Conference, Illinois/EUA.

DENIS, C.; ASSIS, W. O.; COELHO, A. D. Sorting Tomatoes for Industrial Processing Through of Computer Vision System Based on Neural Networks. 2009.In: Proc. of COBEM 2009 - International Congress of Mechanical Engineering, Gramado, RS.

GONZALES, R. C.; WOODS, R. E. Processamento de Imagens Digitais. São Paulo: Ed. Edgard Blücher, 2000.

GUERREIRO, F. S. Dimensionamento e especificação técnica de equipamentos a serem instalados em unidade experimental de tratamento de fluido de perfuração automatizada. Universidade Federal de Uberlândia. Uberlândia/ MG.2012.

KIMMEL, R.; SHAKED, D.; ELAD M.; SOBEL, L. Space-dependent Color Gamut Mapping: A Variational Approach. IEEE Transactions on Image Processing, v. 14, n. 6, p. 796-803, Junho 2005.

LOBATO, F. S., OLIVEIRA-LOPES, L. C., GEDRAITE, R., NEIRO, S. M. S., MURATA, V. V.e SÁ, C. H.M.. Identificação de Modelos para Unidades de Tratamento de Fluido de Perfuração. 2011.In: Anais do ENAHPE - IV Encontro Nacional de Hidráulica de Poços de Petróleo e Gás, Foz do Iguaçu/PR. 